\title{
Application Analysis of AD8479 in the Acquisition Unit of Electronic Current Transformer
}

\author{
Xi Liu, Jianhua Yang, Bifu Qian, Lei Zhang \\ State Grid Wenzhou Electric Power Supply Company, Wenzhou, China
}

\begin{abstract}
Electronic current transformer acquisition unit was installed outdoors and near the circuit breaker. The poor environments, including high common mode voltage, electromagnetic interference and other factors were a serious of disadvantages impacts on the acquisition unit. The paper introduced the parameter characteristics and functions of Rogowski coil, the differential amplifier chip AD8479, and filter circuit in detail. The application circuit was designed based on proper parameters. Lastly, the interference was inhibited effectively, and the electronic current transformer acquisition unit run more reliably resulted from theoretical analysis and field test.
\end{abstract}

\section{Introduction}

Electronic current transformer is one of the most important equipment in intelligent power substation. The output signal of acquisition Unit could be processed by the acquisition unit of it, such as conditioning effect, integral and sample output. The performance of acquisition unit determines the overall accuracy of the transformer, also determines its transient response and reliability. [1-4]

The VFTO (Very Fast Transient Over-voltage, VFTO) stemming from switch operations and ground faults could seriously affect the normal operation of the collection unit, which is the important guarantee of power grid security.

With the development of modern smart power grid and smart substation, electronic current transformers based on Rogowski coil are increasingly installed in GIS (Gas Insulated Switchgear) equipment. The VFTO and TEV (Transient Enclosure Voltage, TEV) and electromagnetic interference generated when the isolating switches are operated. Although the Rogowski coil of acquisition unit is equipped with the protection and filter circuit, it still be interfered by high common mode interference voltage and wide temperature. The efficiency of collecting device is greatly reduced because of the interference, affecting the operation condition of GIS equipment. [5-8]

To solve this key problem, this paper focuses on the working principle of ADI's differential amplifier AD8479, and the design of the corresponding filter circuit and power supply are stated. The circuit designed in this paper can effectively suppress interference and ensure the efficient and stable operation of the equipment.

\section{Electronic current transformer based on Rogowski coil}

Current transformer uses Rogowski coil as a current sensing unit, which is hollow coils of a special structure. Because it has lots of good features such as large measuring electric current pulse amplitude, frequency band width, no core saturation phenomenon, high linearity and good electrical insulation performance, it has been widely applied in the condition of power system fault transient and pulse power technology application field.

The Rogowski coil is coiled evenly around the ring section of nonmagnetic skeleton. The hollow inductor coil is made. It can efficiently isolate high voltage circuits. Its working principle is shown as Fig.1. the e(t) is the secondary side induction voltage, that is the differential voltage of the output signal. The output voltage is proportional to the rate of change of the measured current. [9-13]

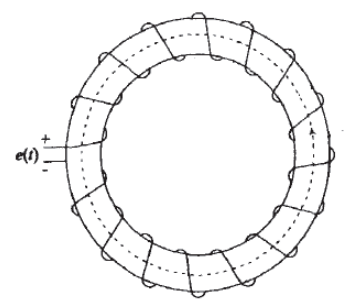

Figure 1. Principle of Rogowski coil 


$$
e(t)=-M \frac{d i}{d t}
$$

In (1), M is the mutual inductance between the bus and the coil, the ' $\mathrm{i}$ ' is the bus-bar current

\section{Differential amplifier AD8479}

Extracting the weak signal from the large common mode noise source, instrument amplifier are used making of two or three operational amplifiers, although the instrument amplifier has excellent common mode rejection ratio, but it requires that the input voltage range is always smaller than the power supply voltage, and cannot cope with the condition of the signal source greater than the power supply voltage or the signal superimposed on the high common mode voltage. And high common mode voltage precision differential amplifier AD8479 Apply to it, it is a precision differential amplifier, it has high input common-mode voltage range, accurate measurement difference signal can be obtained in the highest $\pm 600 \mathrm{~V}$ high commonmode voltage, it also could provide the highest $\pm 600 \mathrm{~V}$ to input common-mode or difference of transient voltage protection. The following characteristics are its advantages: low dissonance voltage, low offset voltage drift, low gain drift, low co-mode rejection, and excellent CMRR (Common Mode Rejection Ratio, CMRR) in a wide frequency range. The internal principle of the AD8479 chip is shown below Fig.2.

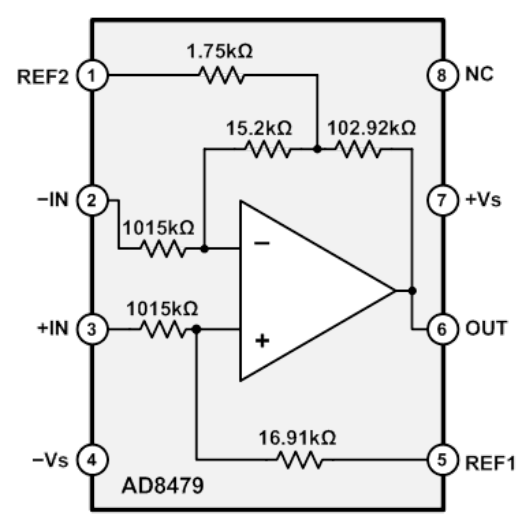

Figure 2. Schematic diagram of AD8479

\section{CMRR of AD847}

When the isolation switch in GIS equipment is operated, the VFTO has a high value and can even reach the base value of 3 times. The Wave-front steep time is low to several ns, frequency is very high. TEV and electromagnetic interference can affect the measurement of the output signal of Rogowski coil

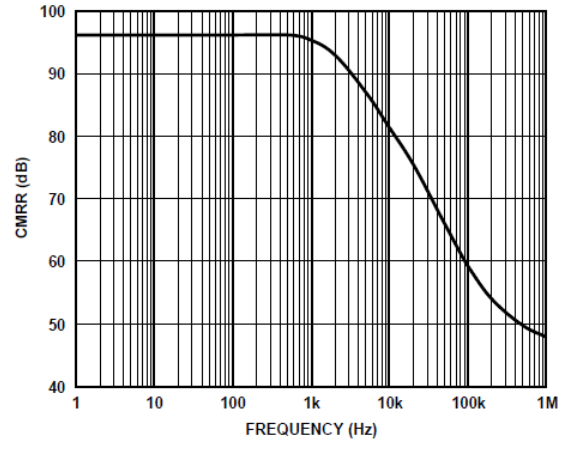

Figure 3. The relationship between CMRR and frequency of AD8479

In the high frequency region of the Fig.3, the performance of the CMRR of AD8479 decreases sharply with the increase of frequency. At this time, the CMRR performance of AD8479 can be significantly improved by the co-mode choke coil in the secondary output side of the Rogowski coil. The common mode choke can suppress the common mode noise and inhibit the noise. In order to further restrict the bandwidth of the differential amplifier signals including difference mode and common mode, the RC filter circuit is built between the tube feet of 2 and 3 in AD8479, as shown in figure 4 below.

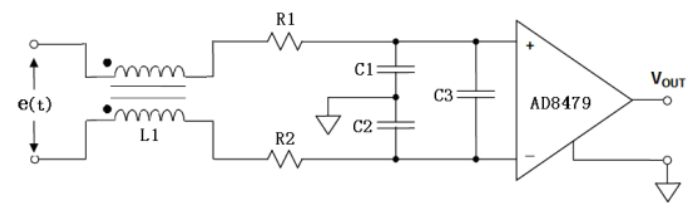

Figure 4. RC filter circuit

In the Fig.4, L1 is the common mode choke coil of RF. For the equilibrium of the differential arm of $\mathrm{AD} 8479, \mathrm{R} 1=\mathrm{R} 2, \mathrm{C} 1=\mathrm{C} 2$ in general. So $-3 \mathrm{db}$ Common mode bandwidth $B W_{C M}$ is:

$$
B W_{C M}=\frac{1}{2 \pi^{*} R 1^{*} C 1}
$$

$-3 \mathrm{db}$ Difference bandwidth $B W_{D I F F}$ is:

$$
B W_{D I F F}=\frac{1}{2 \pi * R 1(2 C 3+C 1)}
$$

Need of special note is, RF common-mode choke coil at high frequencies, such as in a few $\mathrm{MHz}$ to produce apparent impedance, the effect of L1 on the bandwidth of RC filter is not considering in the above (2) and (3). The precise resistances with a temperature coefficient of $15 \mathrm{ppm}$ and $0.1 \%$ accuracy are selected to be R1 and $\mathrm{R} 2$ In the wide temperature range, because the deviation of the differential output of AD8479 is caused by the non-synchronous drift of $\mathrm{R} 1$ and $\mathrm{R} 2$, to ensure the consistency of R1 and R2, R1 and R2 are encapsulated together as a module. The capacitor parameters and the stable NP0 patch capacitors are selected to be $\mathrm{C} 1, \mathrm{C} 2$ and $\mathrm{C} 3$. 


\section{AD8479 power system design}

The design of the differential amplifier AD8479 power supply system is very important. If the design is not properly designed, the input common mode voltage will be reduced, the output signal bandwidth and the pendulum will be affected, and the performance will decrease. As shown in fig.5, the common mode voltage of $\mathrm{AD} 8479$ can reach $600 \mathrm{~V}$ with the two power supplies of $\pm 15 \mathrm{~V}$. In the case of plus or minus $5 \mathrm{~V}$, the common mode voltage is less than $300 \mathrm{~V}$. When only $+5 \mathrm{~V}$ power is supplied, its common mode voltage is less than $230 \mathrm{~V}$.

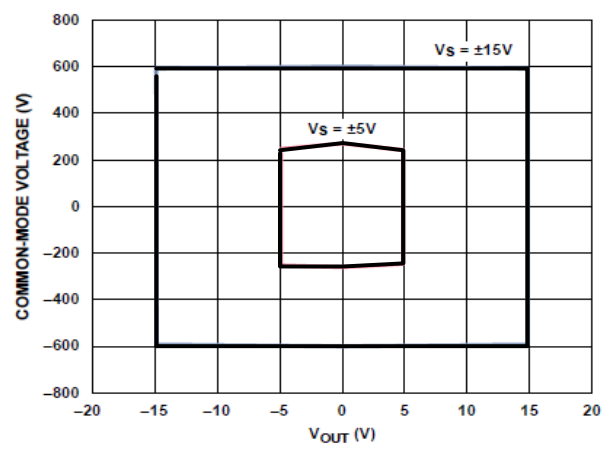

Figure 5. The relationship between the common mode voltage and the output voltage

The power pin effecting on the input terminal of AD8479 could not be ignored. Any noise and coupling that exists on the power line may have an effect on the output signal. As shown in figure 6, when PSRR (Power Supply Rejection Ratio, PSRR) is equal to $20 \mathrm{~dB}$ and $300 \mathrm{k} \mathrm{Hz}$. PSRR is almost zero. The ripple or noise on the power supply is directly reflected in the output terminal. Therefore, in this paper, the application of plus or minus $15 \mathrm{~V} \mathrm{DC} / \mathrm{DC}$ power supply chip is done. And the low dropout regulator is designed after the output end. It can provide AD8479 with relatively clean power supply.

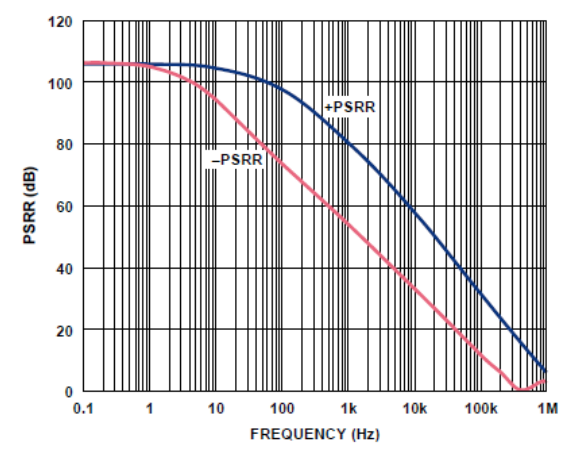

Figure 6. The relationship between PSRR and frequency

\section{Noise analysis}

A In the high common mode voltage, AD8479 detects the small signal of the electronic current transformer output based on Rogowski coil. There is a two-order low-pass passive filter between the output of AD8479 and the ADC Successive approximation chip AD7606 of 16-bit in Fig. 7. [14-18]

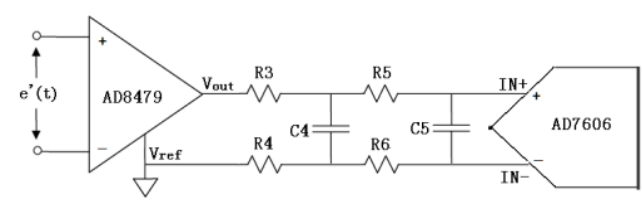

Figure 7. AD7606 Drive circuit diagram

In practical application, the equivalent relationship between the $-3 \mathrm{db}$ cut-off frequency of the filter $f_{-3 d B}$ and the effective noise bandwidth of the loop $B W_{n}$ can be obtained by the proper selection of the components of R3, R4, R5, R6, and C4 and C5, showed as below:

$$
B W_{n}=f_{-3 d B} * k_{n}
$$

$k_{n}$ is the noise bandwidth conversion factor.

$f_{-3 d B}=2 \mathrm{kHz} ; k_{n}=1.22$ because the filter has 2 poles.

The noise analysis of the AD8479 application circuit consists of three parts: voltage noise, current noise and resistance noise. The calculation formula is as follows:

$$
E_{n_{-} i n}=\sqrt{E_{n_{-} v}^{2}+E_{n_{-} i}^{2}+E_{n_{-} r}^{2}}
$$

$E_{n_{-} i n}$ is input noise;

$E_{n_{-} v}$ is voltage noise;

$E_{n_{-} i}$ is current noise;

$E_{n_{-} r}$ is resistance noise.

Since the Gain of AD8479 is fixed to 1, the RMS noise at the output terminal is $E_{n_{-} \text {out }}=E_{n_{-} \text {in }}$ :

$$
E_{n_{-} \text {out }}=E_{n_{-} \text {in }} * \text { Gain }
$$

The estimated peak noise of the output about is $E_{n_{-} \text {out_pp }}$ :

$$
E_{n_{-} \text {out_pp }}=E_{n_{-} \text {out }} * 6
$$

Through the analysis of Fig.4 and Fig.7, combined with the voltage spectrum density curve shown in Fig.8, the formula (4), (5), (6) and (7) can be used to estimate the output $E_{n_{-} \text {out } p p p}$ of $\mathrm{AD} 8479$ about is $30 \mathrm{uV}_{p-p}$. The application of electronic current transformer can be fully satisfied in theory.

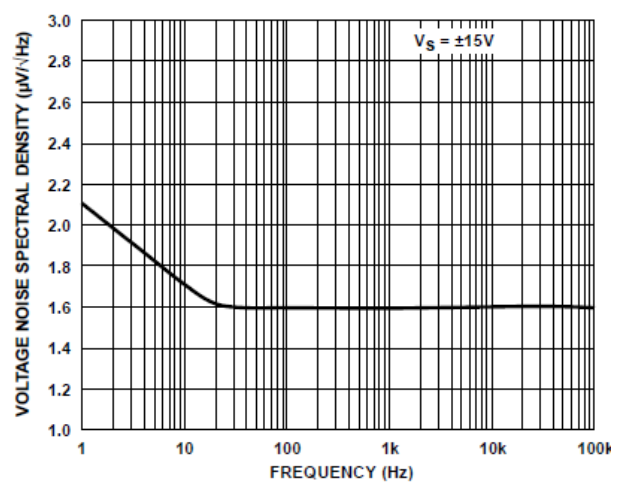

Figure 8. The relationship between voltage spectrum density and frequency

\section{Experiment demonstrates}

Taking the product of Guodian Nanjing Automation CO., Ltd in Xi 'an High Voltage Apparatus Research institute 
Co,. Ltd, testing differential amplifier chip AD8479 in $363 \mathrm{kV}$ high voltage intelligent GIS disconnector opening bus charging current test process, although acquisition cell interference signal port voltage was up to $10 \mathrm{kV}$, frequency reaches $1-10 \mathrm{MHz}$, acquisition unit could still work normally.

From the Fig.9, the recording of charging current test waveforms showed the reliability. Channel 1 and 2 represented the bus voltage in the closed isolation switch, the voltage waveform was the step shape, which was the typical VFTO waveform; Channel 3 and 4 were the waveform of protection current (that was, the output of Rogowski coil). The noise was effectively suppressed, and the signal waveform collected by AD8479 was not distorted and has no large value, which conforms to the requirements of the test specification. Channel 5 mean measuring coil current waveform.

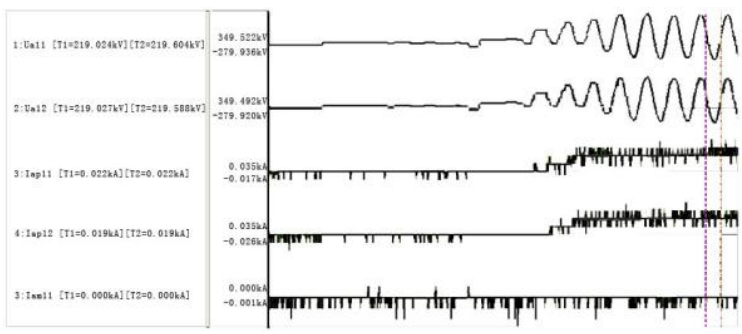

Figure 9. Testing waveforms

\section{Conclusion}

As the core part of electronic current transformer, the normal working state of acquisition unit was the basic guarantee of stable and reliable operation of the whole power system. The method of using the differential amplifier AD8479 chip to extract the small signal from the secondary side of the differential amplifier AD8479 chip was adopted in the paper. It proved that this kind of application completely meets the requirements of electromagnetic interference, precision, transient response and so on.

\section{References}

1. C. Y. Zhang, C. X. Zhang, X. X. Wang, et al. Signal processing system for digital closed-loop fiberoptic current sensor[J]. Proceedings of the CSEE. 2009, 29(30), pp, 42-46.

2. J. Q Gao, X. G Guo. Discussion on intelligent electric power equipment[J]. High Voltage Apparatus, 2010, 46(12), pp, 1-4.

3. Z. H. Xiao. Study and comment of the optical transformers in power system[J]. Power System Protection and Control, 2014, 42(12): 148-154.

4. H. H. Ye, P. Xu, H. L. Zong, et al. The configuration and switch over of voltage transformer in digital substation[J]. Automation of Electric Power Systems, 2008, 32(24), pp,93-95.

5. Y. M. Li, T. F. Zheng, S. B. Zhou, et al. A realization solution of key links for merging unit in smart substation[J]. Automation of Electric Power Systems. 2013, 37(11), pp, 93-98.

6. H. Z. Wang. Research on the factors of disturbance magnetic field of improved air-core coil current transformer[J]. Development \& Innovation of Machinery \& Electrical Products. 2009, 22(3), pp, 166-168.

7. H. L. Zhang. Study and desgin on the Rogowski coil[D]. Qinhuangdao: Yanshan University, 2006.

8. W. B. Li, C. X. Mao, J. M. Lu, et al. Study of the influence of the distributed capacitance on dynamic property of Rogowski coil[J]. Transactions of China Electrotechnical Society, 2004, 19(6), pp, 12-17.

9. R. Wu, Makinwa, K. A. Huijsing. A chopper current feedback instrumentation amplifier with a $1 \mathrm{MHz}$ noise corner and an AC-coupled ripple reduction loop[J]. IEEE Journal of Solid-State Circuits. 2009, 44(12), pp, 3232-3243.

10. Y. H. Lam, W. H. Ki. CMOS bandgap references with self-biased symmetrically matched currentvoltage mirror and extension of sub-1-V design[J]. IEEE Transactions on Very Large Scale Integration (VLSI) Systems, 2010, 18(6), pp, 857-865.

11. S. B. Liang. Measuring methods of composlte errors on current transformer[J]. Relay, 2005, 33(18), pp, 79-83.

12. K. Xiang. Study on digital output interface of electronic transformer[D]. Wuhan, China, Huazhong Universtity of Science and Technology, 2006.

13. E. Qiao, Z. P. An, C. M. Luo. Rogowski coil used in optical current transformer[J]. Transformer, 2000, 37(5), pp, 7-22.

14. B. P. Lathi. Linear system and signals[M]. Carmicheal, California, USA : Berkeley-Cambridge Press, 1992.

15. W. C. Xie, Y. X. Dai. Technology research of integrator for Rogowski electronic current transducer[J]. Electrical Measurement \& Instrumentation, 2011, 48(5), pp, 10-13.

16. H. Wen, Z. S. Teng, S. Y. Guo. Triangular selfconvolution window with desirable sidelobe behaviors for harmonic analysis of power system[J]. IEEE Transactions on Instrumentation and Measurement, 2010, 59(3), pp, 543-552.

17. H. M. Wang, S. X. Zheng, F. Liu. Optimization design for Rogowski coil of high voltage electronic current transducer[J]. High Voltage Engineering, 2005, 31(9), pp, 5-7.

18. Z. L. Long. Design and performance of miniature Rogowski coil for heavy current measurement[J]. High Voltage Engineering, 2007, 33(7), pp, 79-84. 